University of Nebraska - Lincoln

DigitalCommons@University of Nebraska - Lincoln

USDA National Wildlife Research Center - Staff Publications
U.S. Department of Agriculture: Animal and Plant Health Inspection Service

2012

\title{
A Comparison of Methods for Estimating Raccoon Abundance: Implications for Disease Vaccination Programs
}

James C. Beasley

Purdue University, beasley@srel.uga.edu

William S. Beatty

Purdue University, wsbeatty@purdue.edu

Todd C. Atwood

National Wildlife Research Center, tatwood@usgs.gov

Shylo R. Johnson

National Wildlife Research Center

Olin E. Rhodes Jr.

University of Georgia's Savannah River Ecology Lab, rhodes@srel.uga.edu

Follow this and additional works at: https://digitalcommons.unl.edu/icwdm_usdanwrc

Part of the Life Sciences Commons

Beasley, James C.; Beatty, William S.; Atwood, Todd C.; Johnson, Shylo R.; and Rhodes, Olin E. Jr., "A Comparison of Methods for Estimating Raccoon Abundance: Implications for Disease Vaccination Programs" (2012). USDA National Wildlife Research Center - Staff Publications. 1096.

https://digitalcommons.unl.edu/icwdm_usdanwrc/1096

This Article is brought to you for free and open access by the U.S. Department of Agriculture: Animal and Plant Health Inspection Service at DigitalCommons@University of Nebraska - Lincoln. It has been accepted for inclusion in USDA National Wildlife Research Center - Staff Publications by an authorized administrator of DigitalCommons@University of Nebraska - Lincoln. 


\title{
A Comparison of Methods for Estimating Raccoon Abundance: Implications for Disease Vaccination Programs
}

\author{
JAMES C. BEASLEY, ${ }^{1}$ Department of Forestry and Natural Resources, Purdue University, 195 Marsteller Street, W. Lafayette, IN 47907, USA \\ WILLIAM S. BEATTY, Department of Forestry and Natural Resources, Purdue University, 195 Marsteller Street, W. Lafayette, IN 47907, USA \\ TODD C. ATWOOD, USDA APHIS Wildlife Services, National Wildlife Research Center, Fort Collins, CO 80521, USA \\ SHYLO R. JOHNSON, USDA APHIS Wildlife Services, National Wildlife Research Center, Fort Collins, CO 80521, USA \\ OLIN E. RHODES, Jr., ${ }^{2}$ Department of Forestry and Natural Resources, Purdue University, 195 Marsteller Street, W. Lafayette, IN 47907, USA
}

\begin{abstract}
Accurate estimates of demographic parameters are critical to the management of wildlife populations, including management programs focused on controlling the spread of zoonotic diseases. Rabies managers in the United States Department of Agriculture (USDA) have applied a simple raccoon (Procyon lotor) abundance index (RAI) based on cumulative catch of unique raccoons per unit area to determine vaccine-bait distribution densities. This approach was designed to allow for both the collection of biological samples and to index raccoon abundance to determine bait densities for oral rabies programs. However, postbaiting surveillance data indicate that, on average, only $30 \%$ of raccoons sampled have vaccine induced rabies antibody titers, suggesting that bait densities may not be well calibrated to raccoon densities. We trapped raccoons using both capture-mark-recapture (CMR) and the standard RAI to evaluate the accuracy of the current index-based methodology for estimating raccoon density. We then developed a resource selection function from spatial data collected from radio-collared raccoons to standardize trap placement within the existing RAI protocol, and evaluated the performance of this modified RAI approach relative to CMR for estimating raccoon population size. Both abundance and density estimates derived using the RAI consistently underestimated raccoon population sizes compared with CMR methods. Similarly, although the use of resource selection models to inform trap placement appeared to improve the accuracy of the RAI, the effectiveness of this method was inconsistent because of an inability to account for variance in detection probabilities. Despite the logistical advantages of using indices to estimate population parameters to determine vaccine bait distribution densities, our results suggest that adjustments may be necessary to more accurately quantify raccoon abundance, which should improve the effectiveness of rabies management in the United States. In particular, estimates of detection probabilities are needed to more precisely quantify abundance estimates and ensure appropriate vaccine coverage rates. (c) 2012 The Wildlife Society.
\end{abstract}

KEY WORDS density, mammal, mesopredator, Procyon lotor, rabies, raccoon, resource selection function, vaccine.

Zoonoses pose significant risks to animal and human health, and result in substantial economic and public health burdens (Woolhouse and Gowtage-Sequeria 2005, Cutler et al. 2010). Increasingly, wildlife managers must deal with the specter of zoonotic disease and their potential impacts to wildlife populations (e.g., Rodwell et al. 2001, Miller et al. 2003, Keyser et al. 2005), humans (Rupprecht et al. 1995, Daszak et al. 2000), and livestock (Kuiken et al. 2005). Vaccination of reservoir hosts, which reduces the number of susceptible individuals in a population, has long been the standard approach to control disease in both human and domestic animal populations. Recently, vaccination pro-

Received: 13 May 2011; Accepted: 18 January 2012;

Published: 5 April 2012

${ }^{1}$ E-mail: beasley@purdue.edu

${ }^{2}$ Present address: University of Georgia's Savannah River Ecology Lab, Aiken, SC 29802, USA. grams have shown promise for controlling some diseases in wildlife, including rabies (MacInnes et al. 2001), sylvatic plague (Mencher et al. 2004), and bovine tuberculosis (Roberts 1996). Rabies control programs, in particular, have used the landscape-scale distribution of vaccine-laden baits to control or eliminate rabies over extremely large areas in North America and Europe (Mackowiak et al. 1999, MacInnes et al. 2001). In 2009, for example, the National Rabies Management Program and their cooperators distributed approximately 6.5 million vaccine baits in 15 states in ongoing efforts to halt the spread of raccoon (Procyon lotor)variant rabies (United States Department of Agriculture 2009). Such large-scale vaccine distribution campaigns can be logistically cumbersome and vulnerable to a myriad of spatial, temporal, and environmental issues affecting efficacy (Slate et al. 2005, Sattler et al. 2009). Arguably, once a suitable vaccine is developed, the issues most salient to optimizing a vaccine campaign are those regarding bait 
distribution patterns relative to habitat preferences and abundance of reservoir species.

To ensure optimal oral rabies vaccination coverage, the rate of vaccine bait application must be calibrated to the abundance of the target population. Currently, the standard for estimating raccoon abundance involves capturing, marking, and recapturing individuals, and then using modeling procedures based on capture and recapture probabilities to estimate population size (Pollock et al. 2002). Deriving mark-recapture estimates can be labor-intensive and computationally difficult, often making the wide-scale use of these techniques (e.g., implementation on a national scale) impractical or a significant economic and logistical challenge. To overcome these challenges, the National Rabies Management Program has developed and applied a minimum number-known-alive raccoon abundance index (RAI), recognizing that this approach produces conservative indices to raccoon abundance and may need modification, particularly in areas that support high raccoon densities (Ramey et al. 2008). Indeed, seroconversion rates within bait distribution zones generally average 30\% (Slate et al. 2009), suggesting that inadequate bait densities, which may be exacerbated by competition for baits by Virginia opossums (Didelphis virginiana; Smyser et al. 2010), or deficiencies in the vaccine and/or bait may be contributing to less than desired seroconversion rates among raccoons. Generally, vaccination rates of $50-70 \%$ are considered sufficient to break disease transmission (Tinline et al. 2007, Thulke and Eisinger 2008, Recuenco et al. 2009), underscoring the need to continue to evaluate practical methods of estimating raccoon densities to assist with oral rabies vaccination baiting strategies.

Currently, trap selection sites used to calculate RAI are based on qualitative guidance provided to experienced trappers. This approach does not incorporate estimates of detectability, a deficiency that may result in faulty inferences about the population in question (Diefenbach et al. 2003, Norvell et al. 2003, Farnsworth et al. 2005). Given the wide disparity between ideal (50-70\%) and realized (30\%) vaccination rates, an evaluation of RAI performance is needed. Thus, the goal of our research was twofold. First, we evaluated the performance of the RAI relative to standard gridbased capture-mark-recapture (CMR). Next, we developed a standardized, resource selection function (RSF)-based method for determining trap placement that is practical and realistic for use in agricultural ecosystems to improve RAI performance. We then used our RSF model to inform RAI trap placement and evaluated the performance of the RSFmodified index relative to grid-based CMR.

\section{STUDY AREA}

This study took place within the Upper Wabash River Basin in north-central Indiana, USA. Approximately, $71 \%$ of the land area within the Upper Wabash River Basin was cultivated for agricultural production, with corn and soybeans comprising the primary crops. The remaining forest habitat (predominantly oak-hickory-maple [Quercus-Carya-Acer]) in the basin was highly fragmented, with contiguous forest tracts confined to major drainages where frequent flooding or steep topography made the land unsuitable for crop production. The distribution of forest patch sizes within our study area was dominated by patches $<5$ ha $(72 \%)$; large patches ( $>50 \mathrm{ha}$ ) comprised $<3 \%$ of forest patches within the landscape.

We selected 4 study sites within the Upper Wabash River Basin. Each of these sites was $3 \mathrm{~km}^{2}$ in size and was established following the standard RAI protocol; boundaries were delineated to minimize edge effects, and were approximately square to rectangular in shape. Sites ranged from highly fragmented $(<7 \%$ forested $)$ to predominantly forested (>70\% forested) in an effort to represent the range of landscape configurations present in agricultural ecosystems. During 2009, we selected 1 continuously forested (site B) and 1 fragmented site (site A) to contrast density estimates derived using the standard RAI protocol (National Rabies Management Program 2009) and grid-based CMR methods. In 2010, we added 2 additional sites (1 continuously forested site [site D] and 1 fragmented site [site C]) and trapped all 4 sites using CMR as a baseline measure of raccoon density but, because of logistical constraints, selected 2 sites ( $\mathrm{A}$ and $\mathrm{C}$ ) to be trapped using the RAI protocol and the remaining 2 sites ( $B$ and $D$ ) to be trapped using a spatially informed RAI protocol (SI-RAI, see Methods section below; Table 1). As a result, we had no between-year replication for sites $\mathrm{C}$ and $\mathrm{D}$ and the SI-RAI protocol.

\section{METHODS}

\section{Raccoon Capture and Handling}

Regardless of the trapping protocol used (CMR, RAI, or SIRAI), we immobilized all newly captured raccoons with an intramuscular injection of Telazol (Fort Dodge Animal Health, Fort Dodge, IA) at a dosage of $5 \mathrm{mg} / \mathrm{kg}$ of estimated body mass. We ear-tagged (Monel \#3, National Band and Tag Company, Newport, KY) all captured individuals and collected standard morphological and demographic data. We classified raccoons as juveniles $(1 \mathrm{yr})$, yearlings $(2 \mathrm{yr})$, or adults $(\geq 3 \mathrm{yr})$ based on patterns of tooth wear (Grau et al. 1970). We also tagged and processed young-ofthe-year, but excluded these individuals from all analyses because trapping spanned the time period prior to, and while young-of-the-year were emerging from natal dens. For captured individuals that we had previously processed using an alternate trapping protocol (see below), we recorded the tag number and released them without immobilization. To ensure population estimates were not biased because of misread tags, we cross-referenced all recaptured individuals with a list of tagged animals. Similarly, we used genetic data collected for all sampled raccoons to identify whether any individuals had lost their tags and been assigned as new individuals on a subsequent trapping occasion (see Beasley et al. 2011 for details). All trapping methods conformed to American Society of Mammalogists guidelines (Gannon et al. 2007) as well as Purdue University Animal Care and Use Committee policies (protocol 01-079). 
Table 1. Comparison of raccoon abundance and density estimates derived using 3 different trapping protocols in northern Indiana, USA during 2009 and 2010. Values in parentheses represent 95\% confidence intervals for abundance and density estimates and percent difference between estimates derived from either the raccoon abundance index (RAI) or the spatially-informed RAI (SI-RAI) and estimates derived from capture-mark-recapture (CMR) methods are denoted as $\% \Delta$.

\begin{tabular}{|c|c|c|c|c|c|c|c|}
\hline \multirow[b]{2}{*}{ Study site } & \multicolumn{3}{|c|}{2009} & \multicolumn{4}{|c|}{2010} \\
\hline & CMR & RAI $^{a}$ & $\% \Delta$ & CMR & RAI $^{\mathbf{a}}$ & SI-RAI $^{\mathbf{a}}$ & $\% \Delta$ \\
\hline \multicolumn{8}{|l|}{ A (fragmented) } \\
\hline Effective area trapped & $3.4 \mathrm{~km}^{2}$ & $3 \mathrm{~km}^{2}$ & & $3.4 \mathrm{~km}^{2}$ & $3 \mathrm{~km}^{2}$ & & \\
\hline Abundance & $72.2(54.4-90.1)$ & 48.0 (NA) & -33.5 & $42.5(30.5-54.4)$ & 16.0 (NA) & & -62.3 \\
\hline Density $/ \mathrm{km}^{2}$ & $21.2(16.0-24.5)$ & 16.0 (NA) & -24.7 & $12.5(9.0-16.0)$ & $5.3(\mathrm{NA})$ & & -57.6 \\
\hline \multicolumn{8}{|l|}{ B (continuous) } \\
\hline Effective area trapped & $2.02 \mathrm{~km}^{2}$ & $3 \mathrm{~km}^{2}$ & & $2.02 \mathrm{~km}^{2}$ & & $3 \mathrm{~km}^{2}$ & \\
\hline Abundance & $32.6(24.5-40.7)$ & 25.0 (NA) & -23.3 & $29.5(22.13-36.84)$ & & 12.0 (NA) & -59.3 \\
\hline Density $/ \mathrm{km}^{2}$ & $16.1(12.1-20.2)$ & $8.3(\mathrm{NA})$ & -48.4 & $14.54(11.0-18.2)$ & & 4.0 (NA) & -72.5 \\
\hline \multicolumn{8}{|l|}{$\mathrm{C}$ (fragmented) } \\
\hline Effective area trapped & & & & $2.75 \mathrm{~km}^{2}$ & $3 \mathrm{~km}^{2}$ & & \\
\hline Abundance & & & & $35.7(26.3-45.0)$ & $15.0(\mathrm{NA})$ & & -58.0 \\
\hline Density $/ \mathrm{km}^{2}$ & & & & $13.0(9.6-16.4)$ & $5.0(\mathrm{NA})$ & & -61.4 \\
\hline \multicolumn{8}{|l|}{$\mathrm{D}$ (continuous) } \\
\hline Effective area trapped & & & & $2.72 \mathrm{~km}^{2}$ & & $3 \mathrm{~km}^{2}$ & \\
\hline Abundance & & & & $21.7(15.0-28.5)$ & & 30.0 (NA) & +38.2 \\
\hline Density $/ \mathrm{km}^{2}$ & & & & $8.0(5.5-10.5)$ & & 10.0 (NA) & +25.2 \\
\hline
\end{tabular}

${ }^{a}$ Confidence intervals are not available because population parameters were estimated as the total number of individuals captured within each site.

\section{RAI Estimates}

We estimated raccoon densities following the standard RAI protocol (National Rabies Management Program 2009) for sites $\mathrm{A}$ and $\mathrm{B}$ in 2009 , and $\mathrm{A}$ and $\mathrm{C}$ in 2010 (Table 1). The RAI protocol relies on expert knowledge to guide trap site selection. Given that we had been capturing raccoons in the study area for over 8 years, we considered our knowledge in selecting trap sites to be equivalent to that of National Rabies Management Program trappers. Within each site, we set 50 live traps (Tomahawk Live Trap Co., Tomahawk, WI) baited with Hard-Core ${ }^{\circledR}$ Raccoon Lure \#1 (Wildlife Research Center, Ramsey, MN) and marshmallows at locations likely to capture raccoons. We spaced traps within forested areas to ensure adequate coverage of the entire study area. We trapped each study area for 10 days and left traps in place as long as captures occurred. If no captures occurred for a 3- to 4-day interval, we moved traps to new locations $>30 \mathrm{~m}$ from the previous location. We estimated relative density by dividing the number of unique individuals captured by the study area size $\left(3 \mathrm{~km}^{2}\right)$.

\section{CMR Estimates}

We used traditional grid-based mark-recapture procedures to estimate densities of raccoons in sites A and B in 2009 and in sites A, B, C, and D in 2010. To accomplish this, we established trapping grids throughout each $3-\mathrm{km}^{2}$ study area boundary. Within heavily forested sites, we separated grids by approximately 1 home range (73 ha; Beasley et al. 2007b), whereas we trapped all unique forest patches in fragmented sites. Trapping and handling procedures were identical to those described in Beasley and Rhodes (2008). Briefly, we trapped raccoons using box live traps (Tomahawk Live Trap Co.) baited with commercial cat food. We placed traps in a grid (50-m spacing) within forest patches and pre-baited for 3 nights. Following the pre-baiting period, we opened and checked traps for 10 consecutive nights. The total number of traps per grid varied with forest patch size, with a maximum of 30 traps placed in a single grid. Trapping grids could not always be separated by an entire home range because of the spatial arrangement of forest habitat in our study landscape; as a result, several individuals were captured in 2 disparate trapping grids within a site (particularly the fragmented sites). For individuals captured in $>1$ trapping grid $(<8 \%$ of individuals), we eliminated the capture history from the grid with the fewest numbers of captures to minimize any bias in CMR density estimates. We could not combine capture histories for these individuals across grids because not all grids were opened concurrently within some sites.

To estimate raccoon abundance based on CMR capture histories, we followed the methods outlined in Beasley et al. (2011). Briefly, we modeled abundance using the Huggins closed capture-recapture modeling procedure (Huggins 1989) in Program MARK (White and Burnham 1999). We developed separate models for 2009 and 2010 to remove any bias in inter-annual capture probabilities. We pooled capture histories for all unique individuals captured within the same year (all grids combined) to derive robust estimates of the capture $(p)$ and recapture (c) parameters for the combined data set, but we obtained grid-specific estimates of $N$ by treating each trapping grid as a disparate attribute group in MARK (White 2005). We included both sex and age of raccoons as covariates. We evaluated model fit using a biascorrected version of Akaike's Information Criterion $\left(\mathrm{AIC}_{c}\right)$ and used model averaging to determine final population sizes for all models deviating $\leq 4 \mathrm{AIC}_{c}$ units from the model with the smallest $\mathrm{AIC}_{c}$ value (Burnham and Anderson 2002). We pooled abundance estimates (and associated 95\% CI) for all grids within each site to estimate the overall abundance of raccoons at each site.

Based on MARK estimated population sizes, we estimated densities (with 95\% CI) for each site by dividing population size by the relative effective trapping area of the sites. We 
developed effective trapping areas by overlaying a buffer (e.g., Prange et al. 2003) encompassing an area equal to the average raccoon home range size in our study area (73 ha; Beasley et al. 2007b), centered on the centroid of each trapping grid to account for raccoon movements. We then overlaid and merged trapping grid buffers in ArcGIS (Environmental Systems Research Institute, Inc., Redlands, CA) to estimate the total effective area $\left(\mathrm{km}^{2}\right)$ of CMR trapping grids for each site. Based on cumulative abundance estimates for all trapping grids within each site, we estimated the overall density for each site by dividing the estimate of cumulative abundance by the size of the overall buffer.

\section{Radiotelemetry}

During June 2009, we radio-collared 24 raccoons (12 male, 12 female) throughout site B with very high frequency (VHF) transmitters (Wildlife Materials, Carbondale, IL). We only fitted raccoons $\geq 1$ year old with radio-transmitters and we attempted to distribute collars uniformly throughout the study site. From September 2009 to May 2010, we attempted to locate radio-collared raccoons every 1-2 days using a truck mounted Yagi antenna with an electronic compass (AutoComp 1000, KVH Industries, Middletown, $\mathrm{RI})$. We collected the majority of locations at night, but collected approximately $5 \%$ of locations during the day to incorporate den sites in utilization distributions (UDs).

We triangulated raccoon locations using $\geq 3$ bearings ( $90 \%$ had $\geq 4$ ) collected from telemetry stations along roads following the methods outlined in Beasley et al. (2007b). Briefly, we collected bearings for a single location within a 20-minute period to reduce error associated with animal movement. We discarded bearings that did not intersect any other bearing taken for that individual, as well as all bearings intersecting at $<30^{\circ}$. We imported telemetry bearings into Locate III (Nams 2005) to calculate 95\% maximum-likelihood ellipses and individual point locations. We estimated triangulation error for our telemetry system, calculated from 92 known beacon locations, to be $81 \mathrm{~m}$ $(\mathrm{SE}=4.65$; Beasley et al. 2007b).

Within our study area, raccoons exhibit seasonal variation in habitat selection corresponding to the availability of agricultural food resources (Beasley et al. 2007a). Thus, we selected a subset of locations collected prior to corn maturation (Dec-May) to include in the development of our RSF model to maximize the predictability of our model during the time period that trapping would occur. For all individuals with $\geq 25$ locations collected between December and May, we used the Home Range Tools extension (Rodgers and Carr 1998) in ArcGIS to estimate 95\% (home range) and $25 \%$ (core area) fixed kernel UDs. Core areas were limited to the $25 \%$ UD to ensure only the areas within home ranges with the most concentrated activity (presumably representing the most utilized resources) would be included. We assessed possible bias in home range and core area UD sizes due to differences in the number of locations used among individuals using Spearman-rank correlation tests. To estimate the overall space use of radio-collared raccoons as a function of available habitats in our RSF (see below), we derived an overall 95\% UD for all individuals combined. We used least-squares cross validation to estimate smoothing parameters for all UDs.

\section{RSF Development}

To quantify landscape-level habitat attributes within the Upper Wabash River Basin for inclusion in our RSF model, we used a geographic information system (GIS) database developed from 1998 United States Geological Survey digital orthophotos of 1-m resolution (details of habitat delineations are provided in Retamosa et al. 2008). Wooded streams and other water resources often are difficult to delineate from aerial photos. Thus, we also incorporated the National Hydrography data layer and ground-truthing into our GIS to more accurately define water availability. We were interested in testing the performance of a simple model that could be applied to raccoon trapping efforts in novel landscapes, without increasing costs associated with density estimation; therefore, we did not attempt to characterize fine-scale habitat attributes because of the extensive costs associated with the acquisition of these data.

We selected a used-unused study design to develop our RSF model (Manly et al. 2002) of raccoon core areas. We chose to model core areas because habitat quality within the study area is relatively homogeneous at a coarse scale (i.e., home-range scale) and more detectable at the core area scale (Beasley et al. 2007a,b). To minimize any effects of telemetry error associated with individual point locations and differences in the number of locations among individuals, we generated 75 random points within the 25\% UD for each raccoon (1,275 total) using Hawths Tools (Beyer 2004) in ArcGIS as a measure of used habitats. As an estimate of unused habitat, we generated an additional 1,275 random points within the overall $95 \%$ UD boundary estimated for all individuals combined, excluding areas within individual 25\% UDs. Thus, although unused locations fell within the overall 95\% UD of radio-collared raccoons, for the purpose of this analysis, we considered these locations unused as we were specifically interested in modeling the locations of coreuse areas relative to the remaining $95 \%$ UD area. For each of the 2,550 random points (used and unused), we estimated the distance to habitat features defined in our GIS (forest, agriculture, water, developed, and shrubland habitats) using ArcGIS and designated these values as our habitat variables included in subsequent analyses.

To test for collinearity among each of our habitat variables, we examined tolerance and variance inflation factors using weighted least squares regression, and excluded variables with tolerance scores $<0.4$ from analyses (Allison 1999). We used stepwise logistic regression (used vs. available; PROC LOGISTIC, SAS version 9.1; SAS Institute, Inc., Cary, NC) to identify those habitat variables contributing most significantly to observed patterns of space use. We selected habitat variables for inclusion and subsequent retention into the model at $\alpha=0.15$ and 0.05 , respectively. We tested the fit of our model using the Hosmer-Lemeshow goodness-of-fit test statistic and assessed the ability of our RSF model to predict areas within the landscape of 
concentrated raccoon activity using $k$-fold cross validation with 5 data partitions (Boyce et al. 2002). From these data, we estimated the proportion of used locations correctly classified by our model and evaluated the concordance among data partitions using a Spearman-rank correlation test (Boyce et al. 2002).

\section{SI-RAI Application}

Because we derived our RSF from a heavily forested site (site B), we limited our evaluation of the SI-RAI approach in 2010 to the continuously forested sites (B and D) to maximize the utility of our model. For each of these sites, we generated 10,000 random points within the $3-\mathrm{km}^{2}$ study site boundary and estimated the distance of each point to all habitats retained in our logistic regression model (see above) using ArcGIS. We then estimated the resource selection probability for each random point as $\mathrm{RSF}=\exp \left(\beta_{0}+\beta_{1}\right.$ $\left.x_{1}+\beta_{2} x_{2} \ldots+\beta_{n} x_{n}\right)$, using the coefficients of variables retained in our final logistic regression model (Manly et al. 2002). We ranked RSF values for each random point and selected the top $10 \%$ of points as the best locations to deploy traps. Using these points as a list of potential trap site locations, we randomly selected 50 points as initial trapping locations, attempting to space traps at least $30 \mathrm{~m}$ apart to be consistent with the RAI protocol, and set traps following the standard RAI protocol (see above). We moved traps failing to capture a raccoon after 3-4 days according to the RAI protocol, but new trap locations were restricted to the top $10 \%$ of points identified by our RSF model.

\section{Method Comparison}

To test the hypothesis that abundance and density estimates derived using CMR differed from those estimated from the standard RAI protocol, we used paired-sample $t$-tests in SAS (PROC TTEST, SAS verion 9.1). We developed separate models for abundance and density using paired CMR and RIA estimates for sites A and B from 2009 and from sites A and $C$ in 2010 . We evaluated normal probability plots and Kolmogorov-Smirnov goodness-of-fit statistics to assess normality and we used folded F-tests to assess the assumption of equal variances.

Because we only trapped 2 sites using the SI-RAI protocol, we felt performing statistical tests to evaluate whether abundance or density estimates derived using CMR differed from those estimated from the SI-RAI protocol was inappropriate. Rather, we used confidence intervals associated with CMR estimates to determine whether each of the estimates derived using the SI-RAI protocol fell within the upper and lower confidence limits of CMR estimates.

\section{RESULTS}

\section{RAI Estimates}

We did not observe any evidence of matching genotypes within our dataset, suggesting population estimates were not biased because of tag loss. Across the 4 sites trapped following the standard RAI trapping protocol (sites A and B in 2009 and sites A and C in 2010), we captured 104 raccoons (excluding young-of-the-year) over 2,000 trap nights. The number of unique individuals captured per site (i.e., abun- dance) averaged 26, but was highly variable among sites, ranging from 15 to 48 (Table 1). Density estimates derived from RAI trapping ranged from 5.0 to 16.0 raccoons $/ \mathrm{km}^{2}$ $(\bar{x}=8.70, \mathrm{SD}=5.11 ;$ Table 1$)$.

\section{CMR Estimates}

Across the 6 sites trapped using CMR (sites A and B in 2009 and sites A, B, C, and D in 2010), we captured 210 raccoons (excluding young-of-the-year) over 5,540 trap nights. The number of unique raccoons captured ranged from 19 to 60 among sites, averaging 35 captures per site.

Analyses of capture histories for grid-based CMR trapping in Program MARK produced a single top model for sites trapped during $2009\left(p_{\mathrm{t}}=c_{\mathrm{t} \times \text { sex }}\right)$ and 3 competing models for sites trapped in $2010\left(p_{\mathrm{t}+\text { sex }+ \text { age }}=c_{\mathrm{t}+\text { sex }+ \text { age }}, p_{\mathrm{t}}=c_{\mathrm{t}+\text { sex }}\right.$, $\left.p_{\mathrm{t}+\text { sex }}=c_{\mathrm{t}+\text { sex }}\right)$ where initial capture probabilities $(p)$ and recapture probabilities $(c)$ showed temporal variation $\left({ }_{t}\right)$. Based on abundance estimates derived from our MARK models, point estimates of raccoon densities obtained using grid-based CMR ranged from 8.0 to 21.2 raccoons $/ \mathrm{km}^{2}$ among trapping sites $(\bar{x}=14.51, \mathrm{SD}=4.16$; Table 1$)$.

\section{SI-RAI Application}

Seven raccoons had $<25$ locations and were excluded from all analyses. We collected $\geq 25$ usable radio-locations for 17 individuals ( 7 male, 10 female) between December and May 2009-2010. The number of locations per raccoon ranged from 26 to 92 (total $=1,162, \bar{x}=68.35, \mathrm{SD}=$ 23.36), although Spearman-rank correlation tests indicated that neither home range $\left(r_{\mathrm{s}}=0.24, P=0.34\right)$ or core area $\left(r_{\mathrm{s}}=0.14, P=0.59\right)$ sizes were influenced by the number of locations used to estimate UDs among individuals with $\geq 25$ locations. Therefore, we used all locations collected for each individual to estimate $25 \%$ and $95 \%$ UDs. Fixed kernel home range sizes $(\bar{x}=61.51$ ha, $\mathrm{SD}=45.25)$ were smaller than those typically reported for raccoons in rural landscapes, supporting previous research investigating raccoon space use in agricultural ecosystems (Beasley et al. 2007b). On average, core areas accounted for approximately $8 \%$ of the overall size of home ranges and ranged from 1.94 ha to 16.65 ha in size $(\bar{x}=5.13, \mathrm{SD}=4.29)$.

Tolerance scores for all habitat variables within our weighted least squares regression model were $>0.5$; therefore, we retained all habitat variables for inclusion in our logistic model. Of the 5 habitat variables included in our logistic regression analysis, we retained 3 (distance to agriculture, forest, and water) in the final model, all of which were significant $(P<0.001)$. The resulting model was: $\operatorname{RSF}=\exp (0.6473+0.0041$ (distance to agriculture $)-$ 0.0230 (distance to forest) -0.0034 (distance to water)). Although all 3 variables were highly significant in our model, the strength of individual coefficients varied considerably, with the distance to forest having the strongest influence on raccoon core area locations followed by the distance to water. Overall, our RSF model appeared to have a high degree of predictive power $\left(r_{\mathrm{s}}=0.40, P<0.0001\right)$, with $81 \%$ of used and $58 \%$ of random locations classified correctly. Moreover, the Hosmer-Lemeshow goodness-of-fit test statistic indicated an adequate fit for our model $\left(\chi^{2}=15.02, P=0.06\right)$. 
Within the 2 sites (B and D in 2010) trapped using the SI-RAI trapping protocol, we captured 42 unique raccoons (excluding young-of-the-year) over 1,000 trap nights. Of the 42 individuals, we captured 12 at site $\mathrm{B}$ and 30 at site D (Table 1).

\section{Method Comparison}

Both abundance and density estimates derived using CMR were consistently greater than estimates produced from RAI trapping procedures $\left(t_{1,3}=4.7, \quad P=0.02 ; t_{1,3}=11.3\right.$, $P=0.001$, respectively), suggesting that the current RAI protocol may be underestimating raccoon population sizes (Table 1). Overall, SI-RAI based densities for sites B and D were estimated to be $159 \%$ less and $25 \%$ greater than CMR estimates, respectively (Table 1). Thus, although the use of movement data to inform trap placement appeared to improve capture rates of raccoons relative to RAI (and likely CMR) estimates for 1 site, capture rates actually decreased for the other. Both abundance and density estimates were considerably below the lower confidence limit of the CMR estimate for site B. In contrast, abundance estimates for site D exceeded the upper confidence limit of the CMR estimate, but fell within the $95 \%$ confidence interval for density (Table 1). This discrepancy in the magnitude of difference between estimates of abundance and density reflects the more robust and conservative buffer used to estimate density for CMR relative to index-based estimates.

\section{DISCUSSION}

Despite the widespread use of indices in the management of wildlife species, they often fall short in their ability to accurately estimate population parameters (Eberhardt and Simmons 1987, Anderson 2001). Nonetheless, the use of indices is pervasive in the management and conservation of wildlife populations because of the financial and logistical advantages associated with such methods. The results of our study serve to reinforce the value of validating indices, as both abundance and density estimates derived using the standard RAI-based approach underestimated population sizes compared with grid-based CMR trapping, regardless of the extent of landscape connectivity. Although we compared the performance of 2 indices (RAI and SI-RAI) to a statistical model (CMR), we did not validate the modelbased estimate to a known population size, and therefore were unable to determine the accuracy of CMR-derived estimates. However, a direct test of CMR models is seldom feasible, and numerous simulation studies indicate statistical models generally produce population estimates with reduced bias and greater precision than do indices (e.g., Nichols and Pollock 1983).

Estimates of abundance and density generated by the RAI were, on average, $48 \%$ less than estimates from CMR trapping, and differed by as much as $62 \%$. Furthermore, across all sites, RAI estimates consistently were less than the lower bound of the confidence interval surrounding CMR estimates. Although differences in bait type (CMR: cat food vs. RAI: marshmallow and Hard Core ${ }^{\mathrm{TM}}$ lure) may have been a factor influencing these results, index-based estimates also were found to significantly underestimate raccoon density relative to CMR for a high density population of raccoons in Ohio using a consistent bait type (Ramey et al. 2008). Collectively, these data suggest that the current RAI may be underestimating actual densities of raccoons, which may have played a role in the limited oral rabies vaccination coverage rates observed within bait distribution zones (approx. 30\%; Slate et al. 2009). This finding, coupled with recent research indicating that a significant proportion of oral rabies vaccination baits are consumed by non-target species (Olson et al. 2000, Smyser et al. 2010), thus reducing functional bait density for raccoons, suggests that opportunities exist to improve the effectiveness of rabies management in the United States.

The limited ability of the RAI and other indices to accurately estimate density stems from their inability to incorporate imperfect detection probabilities of animals into abundance estimates (Anderson 2001, Williams et al. 2002). For many species, the assumption that all individuals are captured during an abbreviated sampling event is unrealistic, resulting in an underestimate of true populations sizes. Moreover, abundance estimates derived from indices may not always be reliable because of spatial, temporal, or experimental variance in detectability (Anderson 2001, Rosenstock et al. 2002, Collier et al. 2007). Programs directed at the management of zoonotic diseases are particularly sensitive to the accuracy of population parameters as underestimates of these parameters may limit the success of management programs (Konig et al. 2008), whereas overestimates of abundance may inflate costs and increase the likelihood of conflict with non-target species (Flemming et al. 2000, Campbell et al. 2006). Consequently, managers need to improve the accuracy of population estimates derived for disease management programs without significantly increasing costs. In particular, efforts should be made to incorporate the estimation of detection probabilities into trapping protocols to account for variance in detectability and avoid spurious management decisions (Anderson 2001).

Resource selection functions represent the probability of use for individual resource units within the landscape (Manly et al. 2002), and thus the integration of RSFs into trapping designs should increase detection rates over random trap placement designs. Although the use of resource selection models to inform trap placement has an intuitive appeal, our results are inconclusive with regards to the accuracy of density estimates derived using this technique as density estimates were similar between CMR and SI-RAI methods for 1 site (D), but not the other (B). However, the lack of consistency in the performance of the SI-RAI in our study may reflect behavioral differences due to trap habituation, and subsequently detection probabilities, between the sites rather than an inconsistent performance of the RSF model. For example, subsequent to CMR trapping within site $\mathrm{B}$, we observed a decrease in raccoon captures during SI-RAI trapping within areas of sampling overlap (i.e., locations where traps were set during both CMR and SI-RAI trapping), suggesting a potential behavioral response by raccoons to previous trap exposure at this site may have occurred. 
Moreover, raccoons in site B had been exposed to traps for $\geq 2$ trapping cycles (years), whereas site $\mathrm{D}$ only was trapped in 2010 and thus had trap-naïve populations of raccoons. An inability of the SI-RAI to account for changes in detection probabilities could have significantly underestimated raccoon densities within our habituated site. This is further supported by the decline in concordance between CMR and RAI density estimates for site A following initial trap exposure from 2009 (75\% of CMR estimate) to 2010 (42\% of CMR estimate).

Additional replicates controlling for potential biases in capture probabilities due to variance in trap habituation will be necessary to fully evaluate the performance of spatially informed trap placement models to estimate density. Future work also should aim to assess the effectiveness of this technique in novel landscapes to determine the extent of applying an RSF-based model to guide trap placement in a broader spatial context. The RSF used in the SI-RAI protocol was generated from radiotelemetry data collected from 17 raccoons from a single study site (site B), and future work should include collecting spatial data from more individuals distributed over a broader range of landscape configurations. Based on the similarities in density estimates between CMR and SI-RAI for our trap-naive site, we are optimistic that the use of RSFs to objectively guide trap placement will increase the proportion of individuals captured within a population relative to random or subjective methods of trap placement. However, although improvements in index performance may be useful for some management practices (e.g., trapvaccinate-release programs), this method still suffers from an inability to account for variance in detection probabilities. Thus, the full effectiveness of a RSF-based trapping design will only be realized upon integration into trapping protocols allowing for the quantification of detection probabilities.

\section{MANAGEMENT IMPLICATIONS}

In disease vaccination campaigns, a threshold vaccination rate (e.g., $50-70 \%$ for rabies) is required to break disease transmission cycles (Hethcote 1978, Thulke and Eisinger 2008). Maximizing vaccination rates is dependent on ensuring that a sufficient number of baits are accessible to the target population, which can be impacted by competition for bait among non-target species (Smyser et al. 2010) as well as potentially other factors such as the presence and distribution of attractive anthropogenic foods (Prange et al. 2004). Our work indicated that the RAI underestimated raccoon abundance when compared to CMR. Consequently, RAIcalibrated vaccine bait densities may not always be sufficient to induce adequate herd immunity to control rabies. Despite this limitation, the performance of the RAI was fairly consistent throughout this study, suggesting that calibrating the index based on known population sizes may be feasible. Nonetheless, we suggest the following in order to improve the performance of the RAI. First, select trap locations in a probabilistic rather than subjective manner. Probabilistic sampling will allow for inductive inference from the sample data (i.e., a $3-\mathrm{km}^{2}$ reference grid) to the greater population, the scale at which vaccine baits are typically distributed.
Second, employ a double sampling approach (Eberhardt and Simmons 1987) where grid- or UD-based mark-recapture designs are used to estimate abundance at a few sites and a probability-based index is used on a larger sample of areas. If a linear relationship exists between data collected from the 2 methods, then the index values can be calibrated by that relationship (Eberhardt and Simmons 1987). Finally, provide measures of precision for index values so that decisions regarding the density of baits distributed can be adjusted relative to the uncertainty of abundance estimates.

\section{ACKNOWLEDGMENTS}

This study would not have been possible without the cooperation of the Indiana Department of Natural Resources, as well as the landowners who permitted us access to their land. We also are grateful to the numerous field technicians who assisted with the collection of radio-telemetry and markrecapture data. We thank D. Slate, R. Chipman, and T. Algeo for providing information on the National Rabies Management Program raccoon abundance index. Funding for this research was provided by the USDA-APHISWS National Rabies Management program, USDAAPHIS-WS National Wildlife Research Center, and the Department of Forestry and Natural Resources at Purdue University.

\section{LITERATURE CITED}

Allison, P. D. 1999. Logistic regression using the SAS system: theory and application. SAS Institute Inc., Cary, North Carolina, USA.

Anderson, D. R. 2001. The need to get the basics right in wildlife field studies. Wildlife Society Bulletin 29:1294-1297.

Beasley, J. C., T. L. DeVault, M. I. Retamosa, and O. E. Rhodes, Jr. $2007 a$. A hierarchical analysis of habitat selection by raccoons in northern Indiana. Journal of Wildlife Management 71:1125-1133.

Beasley, J. C., T. L. DeVault, and O. E. Rhodes, Jr. 2007b. Home range attributes of raccoons in a fragmented agricultural region of northern Indiana. Journal of Wildlife Management 71:844-850.

Beasley, J. C., Z. H. Olson, G. Dharmarajan, T. S. Eagan II, and O. E. Rhodes, Jr. 2011. Spatio-temporal variation in the demographic attributes of a generalist mesopredator. Landscape Ecology 26:937-950.

Beasley, J. C., and O. E. Rhodes, Jr. 2008. Relationship between raccoon abundance and crop damage. Human-Wildlife Conflicts 2:248-259.

Beyer, H. L. 2004. Hawth's Analysis Tools for ArcGIS. <www. spatialecology.com/htools>. Accessed 03 Jan 2011.

Boyce, M. S., P. R. Vernier, S. E. Nielsen, and F. K. A. Schmiegelow. 2002. Evaluating resource selection functions. Ecological Modelling 157:281300 .

Burnham, K. P., and D. R. Anderson. 2002. Model selection and multimodel inference: a practical information-theoretic approach. Second edition. Springer-Verlag, New York, New York, USA.

Campbell, T. A., S. J. Lapidge, and D. B. Long. 2006. Using baits to deliver pharmaceuticals to feral swine in southern Texas. Wildlife Society Bulletin 34:1184-1189.

Collier, B. A., S. S. Ditchkoff, J. B. Raglin, and J. M. Smith. 2007. Detection probability and sources of variation in white-tailed deer spotlight surveys. Journal of Wildlife Management 71:277-281.

Cutler, S. J., A. R. Fooks, and W. H. M. van der Pool. 2010. Public health threat of new, reemerging, and neglected zoonoses in the industrialized world. Emerging Infectious Diseases 16:1-7.

Daszak, P., A. A. Cunningham, and A. D. Hyatt. 2000. Emerging infectious diseases of wildlife: threats to biodiversity and human health. Science 287:443-449.

Diefenbach, D. R., D. W. Brauning, and J. A. Mattice. 2003. Variability in grassland bird counts related to observer differences and species detection rates. Auk 120:1168-1179. 
Eberhardt, L. L., and M. A. Simmons. 1987. Calibrating population indices by double sampling. Journal of Wildlife Management 51:665-675.

Farnsworth, M. L., L. L. Wolfe, N. T. Hobbs, K. P. Burnham, E. S. Williams, D. M. Theobald, M. M. Conner, and M. W. Miller. 2005. Human land use influences chronic wasting disease prevalence in mule deer. Ecological Applications 15:119-126.

Flemming, P. J. S., D. Choquenot, and R. S. Mason. 2000. Aerial baiting of feral pigs (Sus. scrofa) for the control of exotic disease in the semi-arid rangelands of New South Wales. Wildlife Research 27:531-537.

Gannon, W. L., R. S. Sikes, and the Animal Care and Use Committee of the American Society of Mammalogists. 2007. Guidelines of the American Society of Mammalogists for the use of wild mammals in research. Journal of Mammalogy 88:809-823.

Grau, G. A., G. C. Sanderson, and J. P. Rogers. 1970. Age determination of raccoons. Journal of Wildlife Management 34:364-372.

Hethcote, H. W. 1978. An immunization model for a heterogeneous population. Theoretical Population Biology 14:338-349.

Huggins, R. M. 1989. On the statistical analysis of capture-recapture experiments. Biometrika 76:133-140.

Keyser, P. D., D. C. Guynn, and H. S. Hill. 2005. Population densityphysical condition relationships in white-tailed deer. Journal of Wildlife Management 69:356-365.

Konig, A., T. Romig, C. Janko, R. Hildenbrand, E. Holzhofer, Y. Kotulski, C. Ludt, M. Merli, S. Eggenhofer, D. Thoma, J. Vilsmeier, and D. Zannantonio. 2008. Integrated-baiting concept against Echinococcus multilocularis in foxes is successful in southern Bavaria, Germany. European Journal of Wildlife Research 54:437-447.

Kuiken, T., F. A. Leighton, R. A. M. Fouchier, J. W. LeDuc, J. S. M. Peiris, A. Schudel, K. Stohr, and A. D. M. E. Osterhaus. 2005. Pathogen surveillance in animals. Science 309:1680-1681.

MacInnes, C. D., S. M. Smith, R. R. Tinline, N. R. Ayres, P. Bachmann, D. G. Ball, L. A. Calder, S. J. Crosgrey, C. Fielding, P. Hauschildt, J. M. Honig, D. H. Johnston, K. F. Lawson, C. P. Nunan, M. A. Pedde, B. Pond, R. B. Stewart, and D. R. Voigt. 2001. Elimination of rabies from red foxes in eastern Ontario. Journal of Wildlife Diseases 37:119132 .

Mackowiak, M., J. Maki, L. Motes-Kreimeyer, T. Harbin, and K. Van Kampen. 1999. Vaccination of wildlife against rabies: successful use of a vectored vaccine obtained by recombinant technology. Advances in Veterinary Medicine 41:571-583.

Manly, B. F. J., L. L. McDonald, D. L. Thomas, T. L. McDonald, and W. P. Erickson. 2002. Resource selection by animals: statistical design and analysis for field studies. Second edition. Kluwer Academic Publishers Boston, Massachusetts, USA.

Mencher, J. S., S. R. Smith, T. D. Powell, D. T. Stinchcomb, J. E. Osorio, and T. E. Rocke. 2004. Protection of black-tailed prairie dogs (Cynomys ludovicianus) against plague after voluntary consumption of baits containing recombinant raccoon poxvirus vaccine. Infection and Immunity 72:5502-5505.

Miller, R., J. B. Kaneene, S. D. Fitzgerald, and S. M. Schmitt. 2003. Evaluation of the influence of supplemental feeding of white-tailed deer (Odocoileus virginianus) on the prevalence of bovine tuberculosis in the Michigan wild deer population. Journal of Wildlife Diseases 39:84-95.

Nams, V. O. 2005. Locate III User's Guide. Pacer Computer Software. Tatamagouche, Nova Scotia, Canada.

National Rabies Management Program. 2009. Density index to raccoon population and abundance. United States Department of Agriculture, APHIS, Wildlife Services, Concord, New Hampshire, USA.

Nichols, J. D., and K. H. Pollock. 1983. Estimation methodology in contemporary small mammal capture-recapture studies. Journal of Mammalogy 64:253-260.

Norvell, R. E., F. P. Howe, and J. R. Parrish. 2003. A seven year comparison of relative abundance and distance sampling methods. Auk 120:10131028.

Olson, C. A., K. D. Mitchell, and P. A. Werner. 2000. Bait ingestion by free-ranging raccoons and nontarget species in an oral rabies vaccine field trial in Florida. Journal of Wildlife Diseases 36:734-743.

Pollock, K. H., J. D. Nichols, T. R. Simons, G. L. Farnsworth, L. L. Bailey, and J. R. Sauer. 2002. Large scale wildlife monitoring studies: statistical methods for design and analysis. Environmetrics 13:105-119.
Prange, S., S. D. Gehrt, and E. P. Wiggers. 2003. Demographic factors contributing to high raccoon densities in urban landscapes. Journal of Wildlife Management 67:324-333.

Prange, S., S. D. Gehrt, and E. P. Wiggers. 2004. Influences of anthropogenic resources on raccoon (Procyon lotor) movements and spatial distribution. Journal of Mammalogy 85:483-490.

Ramey, P. C., B. F. Blackwell, R. J. Gates, and R. D. Slemons. 2008. Oral rabies vaccination of a northern Ohio raccoon population: relevance of population density and prebait serology. Journal of Wildlife Diseases 44:553-568.

Recuenco, S., M. Eidson, B. Cherry, and G. Johnson. 2009. Risk-based cost modeling of oral rabies vaccination interventions for raccoon rabies. Zoonoses and Public Health 56:16-23.

Retamosa, M. I., L. A. Humberg, J. C. Beasley, and O. E. Rhodes, Jr. 2008. Modeling wildlife damage to crops in northern Indiana: relative influence of landscape composition and configuration. Human-Wildlife Conflicts 2:225-239.

Roberts, M. G. 1996. The dynamics of bovine tuberculosis in possum populations, and its eradication or control by culling or vaccination. Journal of Animal Ecology 65:451-464.

Rodgers, A. R., and A. P. Carr. 1998. HRE: The Home Range Extension for ArcView. Ontario Ministry of Natural Resources, Centre for Northern Forest Ecosystem, Research, Thunder Bay, Ontario, Canada.

Rodwell, T. C., I. J. White, and W. M. Boyce. 2001. Evaluation of population effects of bovine tuberculosis in free-ranging buffalo (Syncerus caffer). Journal of Mammalogy 82:231-238.

Rosenstock, S. S., D. R. Anderson, K. M. Giesen, T. Leukering, and M. F. Carter. 2002. Landbird counting techniques: current practices and an alternative. Auk 119:46-53.

Rupprecht, C. E., J. S. Smith, M. Fekadu, and J. E. Childs. 1995. The ascension of wildlife rabies: a cause for public health concern or intervention? Emerging Infectious Diseases 1:107-114.

Sattler, A. C., R. A. Krogwold, T. E. Wittum, C. E. Rupprecht, T. P. Algeo, D. Slate, K. A. Smith, R. L. Hale, G. A. Nohrenberg, C. D. Lovell, M. Niezgoda, A. J. Montoney, and R. D. Slemons. 2009. Influence of oral rabies vaccine bait density on rabies sero-prevalence in wild raccoons. Vaccine 27:7187-7193.

Slate, D. E., T. P. Algeo, K. M. Nelson, R. B. Chipman, D. Donovan, J. D. Blanton, M. Niezgoda, and C. E. Rupprecht. 2009. Oral rabies vaccination in North America: opportunities, complexities, and challenges. PLoS Neglected Tropical Diseases 12:e549.

Slate, D., C. E. Rupprecht, J. A. Rooney, D. Donovan, D. H. Lein, and R. B. Chipman. 2005. Status of oral rabies vaccination in wild carnivores in the United States. Virus Research 111:68-76.

Smyser, T. J., J. C. Beasley, Z. H. Olson, and O. E. Rhodes, Jr. 2010. Interspecific competition limits mitigation of wildlife disease: use of Rhodamine B to reveal patterns of bait acceptance. Journal of Wildlife Management 74:1405-1416.

Thulke, H. H., and D. Eisinger. 2008. The strength of 70\%: revision of a standard threshold of rabies control. Developments in Biologicals 131:291-298.

Tinline, R., D. Ball, J. Broadfoot, and B. Pond. 2007. The Ontario rabies model. Queens University, Kingston, Ontario, Canada.

United States Department of Agriculture. 2009. FY 2009 US ORV Distribution Summary. <http://www.aphis.usda.gov/wildlife_damage/ oral_rabies/oral_rabies_info_by_state/us/2009US.pdf $>$. Accessed 03 Jan 2011.

White, G. C. 2005. Correcting wildlife counts using detection probabilities. Wildlife Research 32:211-216.

White, G. C., and K. P. Burnham. 1999. Program MARK: survival estimation from populations of marked animals. Bird Study Supplement 46:120-138.

Williams, B. K., J. D. Nichols, and M. J. Conroy. 2002. Analysis and management of animal populations. Academic Press, San Diego, California, USA.

Woolhouse, M. E., and S. Gowtage-Sequeria. 2005. Host range and emerging and reemerging pathogens. Emerging Infectious Diseases 11:1842-1847.

Associate Editor: Stan Gehrt. 